\title{
Advanced Transcatheter Aortic Valve Implantation (TAVI) Planning from CT with ShapeForest
}

\author{
Joshua K.Y. Swee ${ }^{1}$ and Saša Grbićc ${ }^{1,2}$ \\ 1 Imaging and Computer Vision, Siemens Corporation, Corporate Technology, \\ Princeton, New Jersey, USA \\ 2 Computer Aided Medical Procedures, Technical University Munich, Germany
}

\begin{abstract}
Transcatheter aortic valve implantation (TAVI) is becoming a standard treatment for non-operable and high-risk patients with symptomatic severe aortic valve stenosis. As there is no direct view or access to the affected anatomy, comprehensive preoperative planning is crucial for a successful outcome, with the most important decisions made during planning being the selection of the proper implant size, and determining the correct $\mathrm{C}$-arm angulations. While geometric models extracted from $3 \mathrm{D}$ images are often used to derive these measurements, the complex shape variation of the AV anatomy found in these patients causes many of the shape representations used to estimate such geometric models to fail in capturing morphological characteristics in sufficient detail. In addition, most current approaches only model the aortic valve (AV), omitting modeling the left ventricle outflow tract (LVOT) entirely despite its high correlation with severe complications such as annulus ruptures, paravalvular leaks and myocardial infarction. We propose a fully automated method to extract patient specific models of the AV and the LVOT, and derive comprehensive biomarkers for accurate TAVI planning. We utilize a novel shape representation - the ShapeForest - which is able to model complex shape variation, preserves local shape information, and incorporates prior knowledge during shape space inference. Extensive quantitative and qualitative experiments performed on 630 volumetric data sets demonstrate an accuracy of $0.69 \mathrm{~mm}$ for the AV and $0.83 \mathrm{~mm}$ for the LVOT, an improvement of over $16 \%$ and $18 \%$ respectively when compared against state of the art methods.
\end{abstract}

\section{Introduction}

Valvular heart disease (VHD) is a large subgroup among cardiovascular diseases (CVD) and often requires costly diagnostic, interventional procedures and longterm management [1]. While valvular heart disease has traditionally been treated with surgical repair or replacement, the last several years have seen important advances in concepts, tools, techniques, and patient selection for the treatment of valvular heart disease using non-surgical procedures. Of these procedures, one of the most prevalent is transcatheter aortic valve implantation (TAVI), where

P. Golland et al. (Eds.): MICCAI 2014, Part II, LNCS 8674, pp. 17-24, 2014.

(C) Springer International Publishing Switzerland 2014 

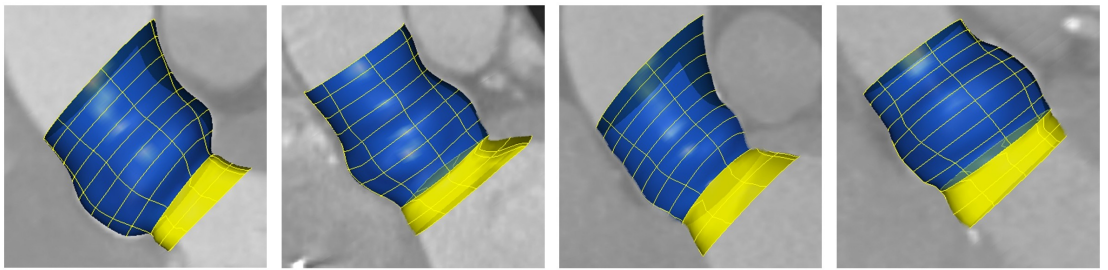

Fig. 1. Examples of complex AV (blue) and LVOT (yellow) variation in patients

a replacement valve is delivered via a catheter. TAVI offers the potential to reduce procedural morbidity and mortality while lowering the treatment cost, and is currently being utilized in non-operable and high-risk surgical patients [2, with likely expansion to intermediate risk patients in the near future [3]. Precise assessment of the aortic valve geometry before this procedure is crucial for a successful outcome, and involves determining the patient specific implant size alongside predicting the accurate $\mathrm{C}$-arm angulation, orthogonal to the annulus of the AV. In current clinical practice, a CT scan is routinely acquired before the procedure in order to determine these parameters manually by the physician.

There have been several proposals to construct geometric models from established diagnostic modalities to assist the physician during pre-operative intervention planning for TAVI. In the context of valvular disease management, the authors in 4 proposed to estimate mitral valve models from MRI. The modeling of the aortic valve from cardiac CT was further investigated in [567]. While models extracted using these methods can provide biomarkers for patient selection and procedure planning, the complex anatomy shape variation among the patient population (as illustrated in Fig. 1) is difficult to model in sufficient detail using standard linear parametric models. In addition, shape variation is often local, with linear parametric shape models unable to preserve local shape detail in the training data if it is not statistically significant. Finally, most current approaches model only the aortic valve geometry, omitting modeling the left ventricle outflow tract entirely despite its high correlation with severe complications such as annulus ruptures, paravalvular leaks and myocardial infarction.

In this paper, we propose a fully automated method to extract patient-specific models of the aortic valve (AV) and the left ventricle outflow tract (LVOT) for TAVI planning using a hierarchical model representation and estimation paradigm. In order to cope with the large shape variability present within the AV and LVOT, we introduce a novel constrained statistical shape model - the ShapeForest - which can model complex shape variation, preserves local shape detail, and incorporates prior information during shape inference. We show that this approach can more accurately approximate shape variation when compared to the classical statistical shape model used in many state of the art methods. Based on these patient-specific modeling techniques, we are able to support and automate the current clinical workflow of TAVI planning, and provide validation through comprehensive clinical experiments backed by a database of 630 
volumetric CT images acquired from several hospitals, covering 536 patients affected by a large spectrum of aortic valve diseases.

\section{Method}

Starting from clinical 3D CT images, a fully automated method is employed in order to segment patient-specific models of the AV and LVOT for use in pre-operative TAVI planning (Fig. 2).

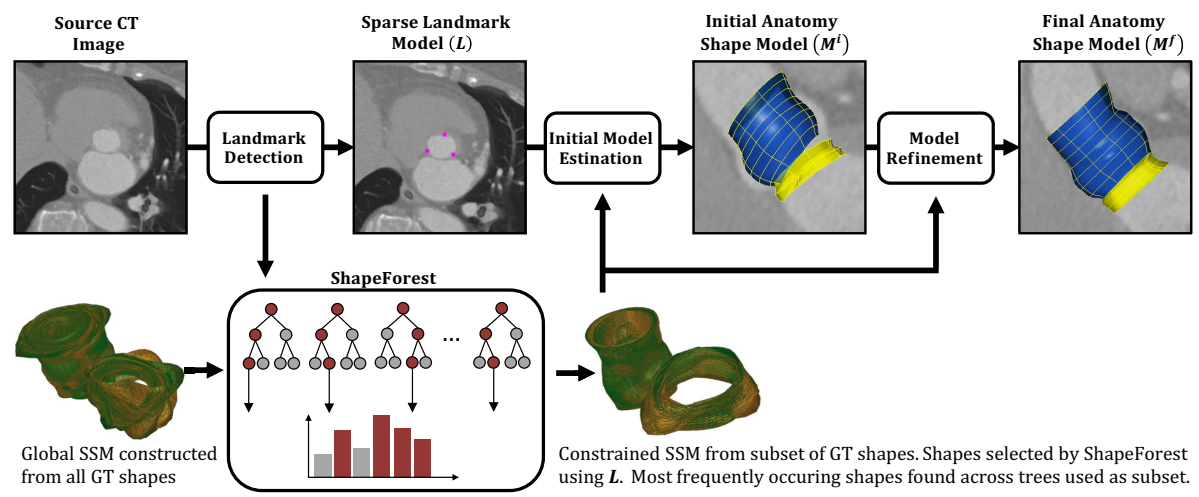

Fig. 2. Method overview, showing the model estimation approach for AV/LVOT sparse landmark models $(L)$ and anatomy shape models $\left(M^{i} / M^{f}\right)$ from clinical CT images.

A hierarchical model representation and parametrization is used for both the AV and LVOT that consists of $L$, the sparse landmark model, and $M$, the anatomy shape model. For the AV, $L_{A V}$ is a set of 6 salient anatomical landmarks (the 3 hinges and 3 commissures of the aortic valve), with $M_{A V}$ being a $3 \mathrm{D}$ surface containing $36 \times 20$ vertices. As the LVOT itself does not possess salient landmarks, we model $L_{L V O T}$ as a set of 7 landmarks taken from nearby anatomical structures (the 3 hinges of the aortic valve, alongside the 2 trigones and 2 commissures of the mitral valve anterior leaflet). Similar to the AV, $M_{L V O T}$ is modelled as a $3 \mathrm{D}$ surface containing $36 \times 10$ vertices. Fig 3 illustrates $L_{A V}$ and $L_{L V O T}$ in detail within $3 \mathrm{D}$ CT images.

To construct the sparse landmark model, we train a position detector for each landmark in $L$ independently. A discriminative learning approach (Probabilistic Boosting Tree) is used, trained with Haar-like features [8] to delineate the points in the images.

The anatomy shape model $M$ is estimated from the sparse landmark model $L$, and the classical statistical shape model (SSM) computed from ground truth anatomy shapes. While the classical SSM (or global SSM/gSSM) is typically represented as a Gaussian parametric model constructed by computing the Principal 


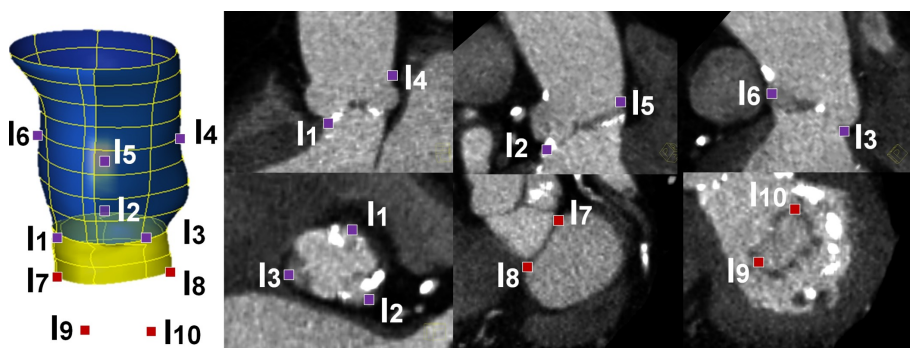

Fig. 3. Sparse Landmark models. $L_{L V O T}=\mathrm{AV}$ hinges $\left(l_{1}, l_{2}, l_{3}\right)$, MV trigones $\left(l_{7}, l_{8}\right)$, MV commissures $\left(l_{9}, l_{10}\right), L_{A V}=\mathrm{AV}$ hinges $\left(l_{1}, l_{2}, l_{3}\right)$, AV comissures $\left(l_{4}, l_{5}, l_{6}\right)$

Component Analysis (PCA) on the covariance matrix of all GPA aligned ground truth shapes $S$, such an approach is unable to accurately model the complex morphological and pathological variation found in the AV and LVOT. We instead employ a constrained form (the constrained SSM/cSSM) wherein the classical SSM is constructed out of $\bar{S}$, the subset of $S$ containing the most representative shapes for each particular instance. We obtain $\bar{S}$ using the ShapeForest.

\subsection{Constrained SSM Construction with ShapeForest}

The ShapeForest infers $\bar{S}$ by learning the shape-manifold based on geometric features defined by the sparse landmark model $L$. Two simple geometric features that were found to be complementary are utilized: distance features and random plane features. Distance features $\left(f_{\text {dist }}\right)$ are generated for each unique landmark pair $(p, q)$ in $L$ as the Euclidean distance between landmarks:

$$
f_{\text {dist }}(p, q)=\sqrt{\sum_{i \in\{x, y, z\}}\left(p^{i}-q^{i}\right)^{2}}
$$

By comparison, random plane features $\left(f_{r p}\right)$ are generated for each landmark $p$ in $L$ as the shortest distance between $p$ and a randomly generated plane:

$$
f_{r p}(p)=\frac{a p^{x}+b p^{y}+c p^{z}+d}{\sqrt{a^{2}+b^{2}+c^{2}}} ; a x+b y+c z+d=0
$$

The ShapeForest itself is constructed as a forest of un-pruned decision trees, similar to Breiman's random forest ensemble classifier [9]. At each tree, each non-leaf node contains a feature $f_{\theta} \in\left\{f_{\text {dist }} \cup f_{r p}\right\}$ and threshold value $\tau$, with both leaf and non-leaf nodes further containing a subset of shapes $S_{t} \in S$. Using this construction, the ShapeForest is able to learn the distance function between the geometric features and the shape variance, clustering shape instances with similar shape characteristics in the leaf nodes. 
Training. A subset of ground truth shapes $S_{t}=\left\{M_{1}, M_{2}, \ldots, M_{v}\right\} \in S$ is randomly sampled at each tree. Using the corresponding set of sparse landmarks $L M_{t}=\left\{L_{1}, L_{2}, \ldots, L_{v}\right\}$, geometric features are computed, and $S_{t}$ and $L M_{t}$ are placed at root nodes in their respective trees. The following training algorithm is executed for every tree in the ShapeForest:

1. For each feature type $f_{\theta} \in\left\{f_{\text {dist }} \cup f_{r p}\right\}$, construct a set of splitting candidates $\phi=\left(f_{\theta}, \tau\right)$, where each $\tau$ represents one of a number of threshold values, equally spaced between $\min \left(f_{\theta}\left(L M_{t}\right)\right)$ and $\max \left(f_{\theta}\left(L M_{t}\right)\right)$.

2. For each $\phi$, partition the set of shapes at the current node $S_{t}$ into left and right subsets:

$$
\begin{aligned}
& S_{l}(\phi)=\left\{s_{v} \mid s_{v} \in S_{t} \wedge f_{\theta}\left(L_{v}\right) \leq \tau\right\} \\
& S_{r}(\phi)=\left\{s_{v} \mid s_{v} \in S_{t} \wedge f_{\theta}\left(L_{v}\right)>\tau\right\}
\end{aligned}
$$

Using Generalized Procrustes Analysis (GPA), align shapes within subsets $S_{t}, S_{l}(\phi)$ and $S_{r}(\phi)$ to produce aligned sets $S_{t}^{a}, S_{l}^{a}(\phi)$ and $S_{r}^{a}(\phi)$.

3. For each $\phi$, compute the information gain $I\left(S_{t}, \phi\right)$ achieved from splitting $S_{t}$ into $S_{l}(\phi)$ and $S_{r}(\phi)$ as:

$$
I\left(S_{t}, \phi\right)=\sum_{s \in S_{t}^{a}} \log (\delta(s))-\sum_{i \in\{l, r\}}\left(\sum_{s \in S_{i}^{a}(\phi)} \log (\delta(s))\right)
$$

Where $\delta(s)=s-\bar{s}$ is the deviation of aligned shape $s$ from mean shape $\bar{s}$, calculated from the set $s$ belongs to $\left(S_{t}^{a}, S_{l}^{a}(\phi)\right.$ or $\left.S_{r}^{a}(\phi)\right)$, similar to [10].

4. Find $\phi^{*}$, the splitting candidate that produces the largest information gain:

$$
\phi^{*}=\underset{\phi}{\operatorname{argmax}} I\left(S_{t}, \phi\right)
$$

5. If $I\left(S_{t}, \phi^{*}\right)$ is greater or equal to a minimum splitting criteria and tree is not at maximum depth, split the node into children, letting $S_{t}=S_{l}\left(\phi^{*}\right)$ at the left node and $S_{t}=S_{r}\left(\phi^{*}\right)$ at the right node. Update $L M_{t}$ at each child node accordingly. Finally, save at current node $S_{t}, f_{\theta}$ and $\tau$ from $\phi^{*}$, and $\bar{L}$ as the GPA-aligned mean of landmarks that are associated with each shape in $S_{t}$.

6. Repeat steps 1 to 5 of the algorithm at each child node until each tree is fully grown.

Testing. When given the sparse landmark model $L$ obtained from a CT image as described earlier in Sect. 2] the ShapeForest computes the feature values for $L, f_{\theta}(L) \in\left\{f_{\text {dist }}(L) \cup f_{r p}(L)\right\}$. Each individual decision tree is then traversed from their root node through the evaluation of $f_{\theta}(L)$ against $\tau$ at each node, branching left or right based on the outcome of this comparison, until a leaf node is reached. The set of shapes $S_{t}$ at each traversed leaf node are finally aggregated in a shape-frequency histogram, with the most frequently occurring shapes found across all trees used to construct an instance specific constrained SSM. 


\subsection{Anatomy Shape Model Estimation}

Using $L$ and the constrained SSM inferred by ShapeForest, an initial shape model $\left(M^{i}\right)$ is generated and fitted to the CT image data. The Powell optimization [11] is then used consecutively to estimate the coefficients for the first 5 largest eigenvectors. Starting with the largest eigenvector the best value is found to accurately match $L$ in the images.

This initial model is then locally refined using an iterative constrained local model (CLM) method [12, whereby feature response images are computed with the probabilistic boosting tree and steerable features. The current shape is iteratively adjusted along its local curvature based on these feature response images, producing the final anatomy shape model $\left(M^{f}\right)$.

\section{$3 \quad$ Experimental Results}

The proposed method was evaluated on a database of $630 \mathrm{CT}$ medical images taken during cardiac cycle end diastole, acquired from several hospitals and covering 536 different patients affected by a large spectrum of aortic valve diseases including severe stenosis and calcifications. Ground truth sparse landmark $(L)$ and anatomy shape $(M)$ models of the AV and LVOT were obtained for all images through manual annotation under the guidance of experts. Experiments were performed on the proposed method (including landmark detection) using three-fold cross validation, with the ShapeForest (using parameters that resulted in best overall performance - 100 trees of depth 20 , each with $30 \%$ of overall training data) constructing constrained SSMs from the top 30\% most frequently occurring shapes.

The model estimation error was first computed for the sparse landmark model (as the mean Euclidean distance between detected landmark points and corresponding ground truth annotations) and anatomy shape model (as the mesh-tomesh error between generated and ground truth meshes). Table 1 summarizes these results for both our proposed method (utilizing cSSMs generated by the ShapeForest), as well as a modification wherein the ShapeForest is not employed and the gSSM is used, as in current state of the art methods. Results of these experiments show our method able improve segmentation accuracy over gSSMgenerated models by $16.8 \%$ for the AV and $18.6 \%$ for the LVOT.

Using the generated models, many clinically relevant measurements can be obtained. 3 measurements of particular importance to TAVI planning were extracted for evaluation - the AV root annulus diameter, alongside LAO/RAO (left/right anterior oblique) and CRAN/CAUD (cranial/caudal) C-Arm viewing angles. Figure 4 shows resultant Bland Altman plots, where a strong correlation between automatically extracted measurements and ground truth is noted.

Finally, an inter-user variability study was conducted in order to gauge the performance of our method relative to the manual performance of experts. Using a subset of 10 randomly selected CT images, we compared the mesh-to-mesh error of the anatomy shape models generated by our method against the manual annotation of four experts. Results of this study are summarized in Fig. 目 
Table 1. Model estimation error results using gSSM (left), and cSSM (right)

\begin{tabular}{|l|c|c|c||c|c|c|c|}
\hline & \multicolumn{3}{|c||}{ gSSM (No ShapeForest) } & \multicolumn{3}{c|}{ cSSM (with ShapeForest) } \\
\hline Model & Mean & STD & Median & Mean & STD & Median & Improv \\
\hline \hline$L_{A V}[\mathrm{~mm}]$ & 2.07 & 1.45 & 1.91 & 2.07 & 1.45 & 1.91 & - \\
$L_{L V O T}[\mathrm{~mm}]$ & 2.20 & 1.11 & 2.01 & 2.20 & 1.11 & 2.01 & - \\
$M_{A V}^{i}[\mathrm{~mm}]$ & 0.99 & 0.50 & 0.85 & 0.80 & 0.33 & 0.67 & $19.1 \%$ \\
$M_{A V}^{f}[\mathrm{~mm}]$ & 0.83 & 0.20 & 0.82 & 0.69 & 0.19 & 0.62 & $16.8 \%$ \\
$M_{L V O T}^{i}[\mathrm{~mm}]$ & 1.65 & 0.61 & 1.51 & 1.33 & 0.58 & 1.24 & $19.4 \%$ \\
$M_{L V O T}^{f}[\mathrm{~mm}]$ & 1.02 & 0.41 & 0.89 & 0.83 & 0.26 & 0.80 & $18.6 \%$ \\
\hline
\end{tabular}
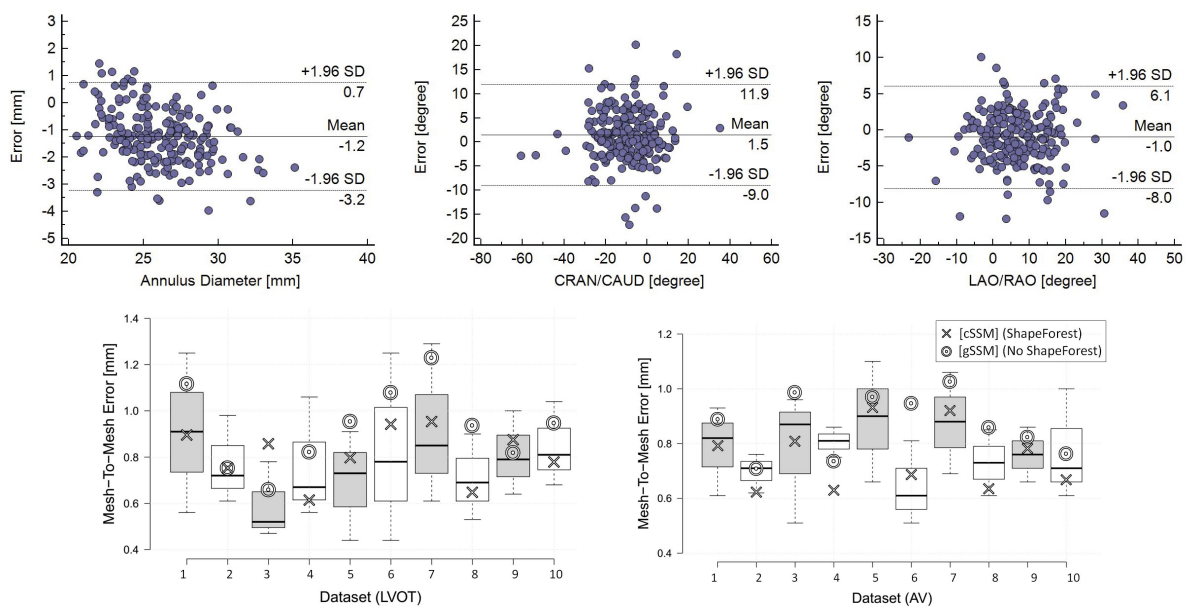

Fig. 4. Top Row: Bland Altman plots of AV root annulus (left), LAO/RAO (center) and CRAN/CAUD angles (right). Bottom Row: Results of the inter-user variability study. Box edges indicate 25th and 75th percentiles of user errors.

showing the system-error relative to the mean measurements of all experts for both the method using constrained SSMs, as well as the global SSM.

Results of this study underscore the importance of the constrained shape representation provided by ShapeForest. Here, results of the proposed method fell within the $75 \%$ inter-user variability range in $95 \%$ of all test cases when constrained SSMs were used. Accuracy when utilizing the global SSM was found to be considerably worse, with results falling within the $75 \%$ inter-user variability range in only $40 \%$ of test cases. Overall, usage of constrained SSMs outperformed the global SSM in $85 \%$ of all cases.

\section{Conclusion}

In this paper we have presented a method for accurate pre-operative planning of TAVI procedures by relying on geometric models of the aortic valve and the left ventricle outflow tract. By utilizing a novel shape representation during shape 
inference with decision trees and geometric features (the ShapeForest), we are able to model complex shape variation, preserve local detail in training data, and incorporate lower-level information in the form of sparse landmarks during shape space learning and shape inference. Validation of our approach through an interuser study and quantitative experiments on $630 \mathrm{CT}$ data sets shows significant improvement when compared to state of the art approaches, and should prove generalizable to other problems that utilize SSMs in conjunction with landmark coordinates, including the geometric modeling of other heart valves.

\section{References}

1. John, D., Buellesfeld, L., Yuecel, S., Mueller, R., Latsios, G., Beucher, H., Gerckens, U., Grube, E.: Correlation of Device landing zone calcification and acute procedural success in patients undergoing transcatheter aortic valve implantations with the selfexpanding CoreValve prosthesis. JACC: Cardiovascular interventions 3(2), 233-243 (2010)

2. Kodali, S.K., Williams, M.R., Smith, C.R., Svensson, L.G., Webb, J.G., Makkar, R.R., Fontana, G.P., Dewey, T.M., Thourani, V.H., Pichard, A.D., Fischbein, M., Szeto, W.Y., Lim, S., Greason, P.S., Malaisrie, S.C., Douglas, P.S., Hahn, R.T., Whisenant, D., Akin, J.J., Anderson, W.N., Leon, M.B.: Two-year outcomes after transcatheter or surgical aortic-valve replacement. NEJM 366(18), 1686-1695 (2012)

3. Reardon, M.J.: Transcatheter aortic valve replacement: Indications and beyond the clinical trials. Texas Heart Institute Journal 40(5), 583-586 (2013)

4. Conti, C.A., Stevanella, M., Maffessanti, F., Trunfio, S., Votta, E., Roghi, A., Parodi, O., Caiani, E.G., Redaell, A.: Mitral valve modelling in ischemic patients: Finite element analysis from cardiac magnetic resonance imaging. In: Computing in Cardiology, pp. 1059-1062 (2010)

5. Ionasec, R.I., Voigt, I., Georgescu, B., Wang, Y., Houle, H., Higuera, F., Navab, N., Comaniciu, D.: Patient-specific modeling and quantification of the aortic and mitral valves from 4-D cardiac CT and TEE. TMI 29(9), 1636-1651 (2010)

6. Waechter, I., Kneser, R., Korosoglou, G., Peters, J., Bakker, N.H., Van Der Boomen, R., Weese, J.: Patient specific models for planning and guidance of minimally invasive aortic valve implantation. In: Jiang, T., Navab, N., Pluim, J.P.W., Viergever, M.A. (eds.) MICCAI 2010, Part I. LNCS, vol. 6361, pp. 526-533. Springer, Heidelberg (2010)

7. Grbic, S., Ionasec, R., Vitanovski, D., Voigt, I., Wang, Y., Georgescu, B., Navab, N., Comaniciu, D.: Complete valvular heart apparatus model from 4D cardiac CT. Medical Image Analysis 16(5), 1003-1014 (2012)

8. Zheng, Y., Georgescu, B., Ling, H., Zhou, S.K., Scheuering, M., Comaniciu, D.: Constrained marginal space learning for efficient $3 \mathrm{~d}$ anatomical structure detection in medical images. In: CVPR, pp. 194-201 (2009)

9. Breiman, L.: Random forests. Machine Learning 45(1), 5-32 (2001)

10. Cootes, T.F., Taylor, C.J., Cooper, D.H., Graham, J.: Active shape models-their training and application. Computer Vision and Image Understanding 61(1), 38-59 (1995)

11. Powell, M.J.: A fast algorithm for nonlinearly constrained optimization calculations. In: Numerical Analysis, pp. 144-157 (1978)

12. Cootes, T.F., Ionita, M.C., Lindner, C., Sauer, P.: Robust and accurate shape model fitting using random forest regression voting. In: Fitzgibbon, A., Lazebnik, S., Perona, P., Sato, Y., Schmid, C. (eds.) ECCV 2012, Part VII. LNCS, vol. 7578, pp. 278-291. Springer, Heidelberg (2012) 